\title{
Understanding Chemical Selectivity through well selected Excited States
}

\author{
F. Guégan, ${ }^{*, \dagger}$ T. Pigeon, ${ }^{\ddagger}$ F. De Proft,, V. Tognetti, ${ }^{\S}$ L. Joubert,,$\S$ \\ H.Chermette, ${ }^{\ddagger}$ P.W. Ayers,,$\|$ D. Luneau,${ }^{\perp}$ and C. Morell ${ }^{*, \ddagger}$ \\ †IC2MP UMR 7285, Université de Poitiers - CNRS, 4, rue Michel Brunet TSA \\ 51106-86073 Cedex 9 Poitiers, France. \\ $\ddagger$ Université de Lyon, Institut des Sciences Analytiques, UMR 5280, CNRS, Université \\ Lyon 1, ENS Lyon - 5, rue de la Doua, F-69100 Villeurbanne, France. \\ E-mail: frederic.guegan@univ-poitiers.fr; christophe.morell@univ-lyon1.fr
}

\section{Introduction}

Explaining, rationalising and predicting molecular reactivity ${ }^{1}$ and $/$ or selectivity has always been a central goal in theoretical chemistry. Many frameworks ${ }^{2,3}$ have been proposed to account for them.

These frameworks can be divided in two categories: either one explicitly models the chemical events, i.e. the displacements of electron density and nuclei as the reaction proceeds, or one focuses on some relevant species, generally the reagents or the reactive intermediates, and tries to understand their reactivity and selectivity through some descriptors ${ }^{4}$ derived from first-principles. These latter descriptors often share the same conceptual basis, relating reactivity and selectivity of a system to the ability of its electron cloud to distort during a chemical event.

Excitability, on the other hand, describes the propensity of a system to reach its excited 
states. Excitations which lead to well separate the electrons through space are expected to be associated with low transition energies - this is for instance used to account for the lower first excitation energy of azulene compared to naphtalene. ${ }^{5}$ As such, one may state (with all due care) that the more the electron density of a system is easily distorted in an valence excited state, the lower in energy the excited state is. This "relocation rule" is for instance used in the development of photoactive systems (push-pull molecules...) and in the rationalization of the selectivity of a reaction performed at a specific excited state.

Thus, a connection between excitability and reactivity seems to exist, since both rely on how easily the electron cloud is modified under an external perturbation. In other words, excitability and reactivity may be connected through polarizability. Interestingly, this formal connection was firstly proposed in the 1940 's by Walsh. ${ }^{6}$ By comparing the longest absorption wavelength of ethylene oxide $(1850 \AA)$ with that of ethylene and of ethane, Walsh stated that the former compound contains electrons that are as loosely bound as that of ethylene, compared to the electrons of ethane that seem more tightly bound. More recently, through a wide set of chemical processes, Pearson ${ }^{7}$ correlated the reactivity of a molecule with its highest wave number absorption, the general rule of thumb being the higher the wave number the more stable the molecule. This was an early and first formulation of the Maximum Hardness Principle. ${ }^{8}$ Even though the rule suffers from some exceptions, Pearson showed how to predict which molecules are not complying with the general rule and provided some ways to avoid pitfalls.

Yet, very few developments have explicitly followed Walsh and Pearson's hypothesis, even though most reactivity descriptors can actually be linked to excitability. For instance, this is rather plain for Pearson's Hardness, since a proposal to assess it using the first excitation energy has been made by Nagy a while ago. ${ }^{9}$ Another example is the frontier molecular orbitals theory development, ${ }^{10}$ since within the frozen orbital scheme, the lowest excitation arises from the promotion of one electron from the HOMO to the LUMO (note that this was already pointed out by Pearson in his 1988 publication). This is also true for the dual 
descriptor, as highlighted by the state-specific development of Tognetti et al. ${ }^{1112}$

In this publication, we propose a new set of reactivity and selectivity descriptors derived within a Rayleigh-Schrödinger perturbation theory framework, thus involving explicitly the excited states of the system under study. In a first part, the working equations for these descriptors are derived, in the case of a perturbation in the external potential $\delta v(\mathbf{r})$. Then, arguments to impart explicit chemical meaning to these descriptors are put forward. In a second part, we then illustrate the efficiency of these descriptors on four different chemical systems.

\section{Energy and electron density responses}

Suppose we know the whole set of solutions of the stationary electronic Schrödinger equation (spectrum and wavefunctions) for a given chemical system:

$$
\mathcal{H}|n\rangle=E_{n}|n\rangle
$$

$\mathrm{n}$ indexing the eigenstates, starting from $n=0$ for the ground state, $n \geq 1$ referring to excited states. Let $|0\rangle$ be the eigenvector of the non-degenerate ground state, and $\mathcal{H}_{\text {pert }}$ the Hamiltonian of a time-independent perturbation applied on the system.

The perturbed ground-state wavefunction $|\psi\rangle$ is given at first order in the RayleighSchrödinger ${ }^{13}$ formalism by

$$
\begin{aligned}
|\psi\rangle & =c_{0}\left(|0\rangle+\sum_{k \neq 0} \frac{\left\langle k\left|\mathcal{H}_{\text {pert }}\right| 0\right\rangle}{E_{0}-E_{k}}|k\rangle\right) \\
& =c_{0}\left(|0\rangle+\sum_{k \neq 0} c_{k}|k\rangle\right)
\end{aligned}
$$


with

$$
c_{k}=\frac{\left\langle k\left|\mathcal{H}_{\text {pert }}\right| 0\right\rangle}{\left(E_{0}-E_{k}\right)}
$$

and

$$
\left|c_{0}\right|=\sqrt{\frac{1}{1+\sum_{k \neq 0} c_{k}^{2}}} .
$$

Note already that, due to the Hamiltonian hermiticity, wavefunctions and thus coefficients $c_{k}$ and normalisation constant $c_{0}$ can and will be chosen to be real.

$c_{0} c_{k}$ directly translates the amplitude of excited state $\mathrm{k}$ within the perturbed wave function. Its square is therefore the fraction of electron promoted from the ground state into the state $\mathrm{k}$ in order to polarize the system. The perturbed electron density, $\rho_{\text {pert }}(\mathbf{r})$, is thus simply:

$$
\rho_{\text {pert }}(\mathbf{r})=\langle\psi|\widehat{\rho}(\mathbf{r})| \psi\rangle
$$

with $\widehat{\rho}(\mathbf{r})$ the one-electron density operator, defined for a N-electron system as:

$$
\widehat{\rho}(\mathbf{r})=\sum_{i=1}^{N} \delta\left(\mathbf{r}-\mathbf{r}_{i}\right)
$$

Developing $\psi$ in Equation 6, one gets

$$
\begin{aligned}
\rho_{\text {pert }}(\mathbf{r})= & c_{0}^{2}\langle 0|\widehat{\rho}(\mathbf{r})| 0\rangle+\sum_{l \neq 0} c_{0}^{2} c_{l}\langle 0|\widehat{\rho}(\mathbf{r})| l\rangle \\
& +\sum_{k \neq 0} c_{0}^{2} c_{k}\langle k|\widehat{\rho}(\mathbf{r})| 0\rangle+\sum_{l \neq 0} \sum_{k \neq 0} c_{0}^{2} c_{k} c_{l}\langle k|\widehat{\rho}(\mathbf{r})| l\rangle .
\end{aligned}
$$


Since all wavefunctions are chosen to be real, $\langle 0|\widehat{\rho}(\mathbf{r})| l\rangle=\langle l|\widehat{\rho}(\mathbf{r})| 0\rangle$, thus

$$
\begin{aligned}
\rho_{\text {pert }}(\mathbf{r}) & =c_{0}^{2}\langle 0|\widehat{\rho}(\mathbf{r})| 0\rangle+2 \sum_{k \neq 0} c_{0}^{2} c_{k}\langle k|\widehat{\rho}(\mathbf{r})| 0\rangle+\sum_{l \neq 0} \sum_{k \neq 0} c_{0}^{2} c_{k} c_{l}\langle k|\widehat{\rho}(\mathbf{r})| l\rangle \\
& =c_{0}^{2}\left(\rho_{0}(\mathbf{r})+2 \sum_{k \neq 0} c_{k} \rho_{0}^{k}(\mathbf{r})+\sum_{k \neq 0} \sum_{l \neq 0} c_{k} c_{l} \rho_{k}^{l}(\mathbf{r})\right)
\end{aligned}
$$

where $\rho_{0}(\mathbf{r})$ is the unperturbed ground-state electron density and $\rho_{k}^{l}(\mathbf{r})$ the transition density between states $k$ and $l$. A first simplification should be made: in this expression, the last term is formally a second-order contribution, and should thus be dropped. Assuming furthermore the perturbation is small, $c_{0}$ and $c_{0}^{2}$ should be close to unity, thus:

$$
\rho_{\text {pert }}(\mathbf{r}) \approx \rho_{0}(\mathbf{r})+2 \sum_{k \neq 0} c_{k} \rho_{0}^{k}(\mathbf{r})
$$

As such, the electron density reshuffling at first order under perturbation may be written as

$$
\delta \rho(\mathbf{r})=\rho_{\text {pert }}(\mathbf{r})-\rho_{0}(\mathbf{r}) \approx 2 \sum_{k \neq 0} c_{k} \rho_{0}^{k}(\mathbf{r})
$$

The shifted electron fraction due to a specific perturbation is therefore :

$$
\delta N=\frac{1}{2} \int|\Delta \rho(\mathbf{r})| d^{3} \mathbf{r}
$$

Similarly, the first and second order energy responses are given by

$$
\begin{aligned}
& E^{(1)}=\left\langle 0\left|\mathcal{H}_{\text {pert }}\right| 0\right\rangle \\
& E^{(2)}=\sum_{k \neq 0} \frac{\left|\left\langle k\left|\mathcal{H}_{\text {pert }}\right| 0\right\rangle\right|^{2}}{E_{0}-E_{k}}=\sum_{k \neq 0} c_{k}^{2} \times\left(E_{0}-E_{k}\right)
\end{aligned}
$$

The second formulation of equation 14 helps understanding its meaning. As $c_{k}^{2}$ is the fraction of electron promoted from the ground state into state $|k\rangle$, the quantity $-E^{(2)}=c_{k}^{2}\left(E_{k}-E_{0}\right)$ is 
therefore the energy required for this transition. Hence, $-E^{(2)}$ represents the energy needed to obtain the new electron configuration. If now we further specify that the perturbation is simply a variation in the external potential, $\mathcal{H}_{\text {pert }}=\delta v(\mathbf{r}),{ }^{14}$ we may write that

$$
\begin{aligned}
E^{(1)} & =\int \rho_{0}(\mathbf{r}) \delta v(\mathbf{r}) d^{3} \mathbf{r} . \\
E^{(2)} & =\sum_{k \neq 0} \frac{\left|\int \rho_{0}^{k}(\mathbf{r}) \delta v(\mathbf{r}) d^{3} \mathbf{r}\right|^{2}}{E_{0}-E_{k}} . \\
\delta \rho(\mathbf{r}) & =2 \sum_{k \neq 0} \frac{\int \rho_{0}^{k}\left(\mathbf{r}^{\prime}\right) \delta v\left(\mathbf{r}^{\prime}\right) d^{3} \mathbf{r}^{\prime}}{E_{0}-E_{k}} \rho_{0}^{k}(\mathbf{r}) .
\end{aligned}
$$

As this perturbation only acts upon the spatial variables, only excited states with the same spin symmetry as the ground state need to be computed. It is worth noticing that all terms appearing in equations 11 to 17 can be easily evaluated for a given $\delta v(\mathbf{r})$. Through the computation of the excitation spectrum of a molecule, we may calculate its electron density and energy responses (1st order correction for the electron density, 1st and 2nd corrections for the energy) to any perturbation in the external potential. It is important to emphasize that the numerators of equations 16 and 17 enable the connection between the ground state of the molecule with one of its excited states. It is very likely that some excited states will appear more relevant than others to express the density polarization. Monitoring which excited state contributes most will provide valuable information to predict the fate of the system. Some specific examples of such rationale are given in section 4 .

Besides this, one may wonder about the chemical meaning of these responses.

Electron density reorganisation. The electron density reorganisation is rather selfexplanatory: this quantity simply expresses the way the electron density is distorted in order to accommodate the system to the perturbation. It is important to keep in mind that this electron density reshuffling is evaluated at a fixed molecular geometry; the electrostatic potential created by the nuclei is thus supposed to be unchanged.

Two aspects of the electron density reshuffling may be distinguished: one may either 
focus on the extent of electron density that distorts (number of electrons that are displaced) or on the way these electrons are reorganised (the shape of the electron reshuffling). The electron density variation, as expressed in Equation 17, can be seen a measure of the latter, while the shifted electron fraction (Equation 12) can be used to assess the former.

This electron density response can also be seen as a measure of local polarisability, since it evaluates how easily electrons move when submitted to any perturbation. Further chemical meaning can be retrieved if we recognize that the electron density variation under a change in the external potential $\delta v(\mathbf{r})$ can also be written as

$$
\delta \rho(\mathbf{r})=\int \chi\left(\mathbf{r}, \mathbf{r}^{\prime}\right) \delta v\left(\mathbf{r}^{\prime}\right) d^{3} \mathbf{r}^{\prime}
$$

with $\chi\left(\mathbf{r}, \mathbf{r}^{\prime}\right)$ the linear response kernel (note that if we develop equation 11 with $\mathcal{H}_{\text {pert }}=$ $\delta v(\mathbf{r})$, we retrieve a known formulation for the linear response kernel). ${ }^{15}$ Numerous works revealed the possibility to extract chemical information from the linear response ${ }^{16}$, such as electronic effects ${ }^{17}$ (mesomery and induction), shell structure of atoms but also polarisabilities. ${ }^{18-20}$ Thus, the first order density response is as well expected to convey knowledge about the chemical properties of a system, noticeably reactivity and selectivity.

Energy responses. The first order energy response is also quite plain: since we chose here that $\mathcal{H}_{\text {pert }}=\delta v(\mathbf{r})$, this quantity is simply the electrostatic stabilisation or destabilisation experienced by the electronic system under the perturbation. In the case of a perturbation by a point charge and if one includes the nuclei distribution, this first correction corresponds to the interaction energy of a long-known descriptor, the molecular electrostatic potential $(\mathrm{MEP})^{21}$, with the perturbation. Much attention has already been paid to the MEP, ${ }^{\mathrm{a}}$ and its relation with selectivity. As such we will not explore its chemical meaning much further.

The second order response is on the other hand more original. Obviously, from its

aNoticeably, 13615 references were found containing the concept "Molecular Electrostatic Potential", according to a SciFinder bibliographical survey conducted on September 10, 2019. 
mathematical expression, it will always be a stabilising contribution (negative) in the ground state. It represents the energetic stabilisation experienced by the system by distorting its electron density in response to the perturbation. Said otherwise, it translates the stabilisation energy associated to the electron density polarisation. Note this second order correction to the electronic energy is also well known in conceptual DFT:

$$
E^{(2)}=\frac{1}{2} \iint \chi\left(\mathbf{r}, \mathbf{r}^{\prime}\right) \delta v\left(\mathbf{r}^{\prime}\right) \delta v(\mathbf{r}) d^{3} \mathbf{r} d^{3} \mathbf{r}^{\prime}
$$

Interestingly, the second order response energy strongly depends on the location of the point charge perturbation. It is thus expected to carry quantitative information about the selectivity of a given system.

\section{Computational details}

All DFT and TD-DFT calculations were performed using the 4.0.2 version of ORCA. ${ }^{22}$ Unless stated otherwise, structures were fully optimised in the ground state at the B3LYP level, using all electron triple zeta basis sets (cc-PVTZ in the case of example 4.3, def2TZVP in all other cases). TD-DFT calculations were performed under the Tamm-Dancoff approximation $^{23}$ (default implementation in ORCA), at the same level of theory. In all cases, the first 50 excited states were computed, and the transition densities from the ground state to each of these excited states were extracted in the Gaussian cube format using the orca_plot utility. Benchmarking on a simple example showed that very fine grids should be set to achieve convergence on the calculation, thus a $160 \times 160 \times 160$ grid was employed, corresponding to a mesh of approximately 0.05-0.07 $\AA$ for all considered examples.

A home-made Fortran program was then used to compute the first order electron density reshuffling (in cube format), as well as the first and second order energy corrections. Details about the code will be published elsewhere. It can be obtained, upon request, from the

\footnotetext{
${ }^{\mathrm{b}}$ More exactly, B3LYP as implemented within Gaussian.
} 
authors.

\section{Applications}

\section{Revisiting a textbook SN2 reaction}

In this first example, we wish to explore the early stages of a bimolecular nucleophilic substitution $\left(\mathrm{SN}_{2}\right)$. We here considered the attack of a nucleophile on the prototype reagent $\mathrm{CH}_{3} \mathrm{Cl}$. It is known to proceed via backside attack of the $\mathrm{C}-\mathrm{Cl}$ bond, and is expected to lead to a pentacoordinate $\mathrm{C}$ in the transition state structure (Walden inversion). ${ }^{24}$

Usually, this feature is explained on the basis of MO theory: the lowest unoccupied MO is an antibonding $\mathrm{C}-\mathrm{Cl} \sigma^{\star}$, which develops along the $\mathrm{C}-\mathrm{Cl}$ axis, principally on the carbon atom and outwards of the molecule. Interaction with a filled MO on the nucleophile is thus maximal under a backside attack, and should lead to the simultaneous formation of a C-nucleophile bond and breaking of the $\mathrm{C}-\mathrm{Cl}$ bond, because of electron transfer in the antibonding $\sigma^{\star} \mathrm{C}-\mathrm{Cl} .{ }^{25}$

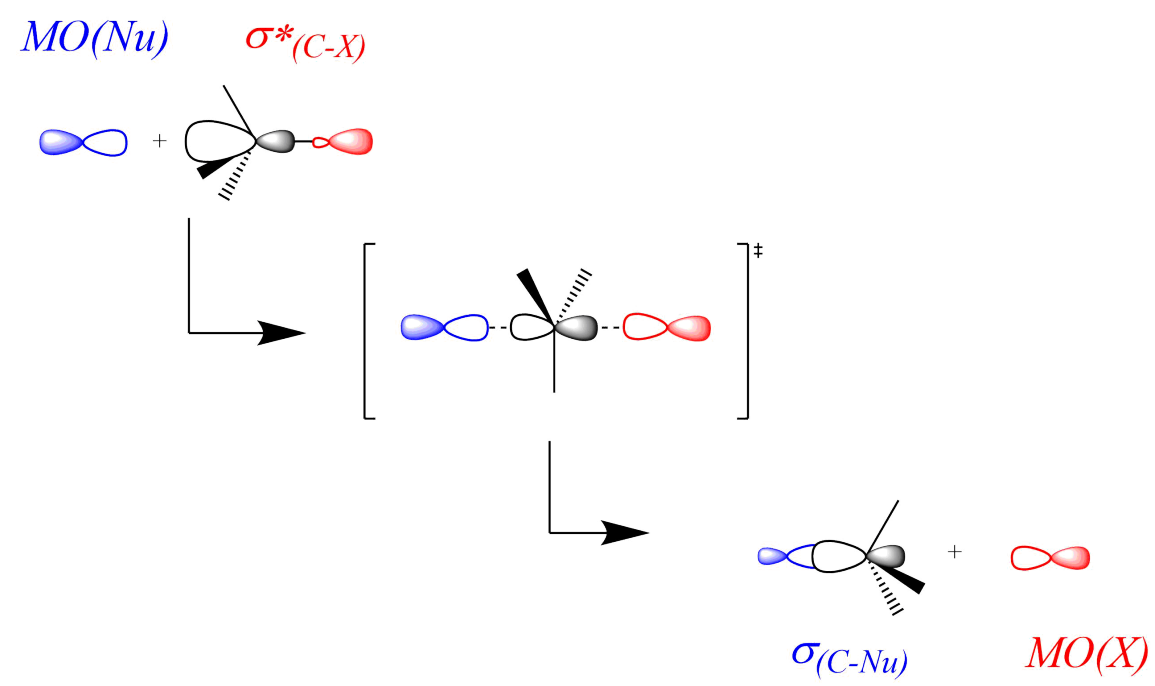

Figure 1: Schematic representation of the Frontier Molecular Orbital interpretation of the $\mathrm{SN}_{2}$ reaction mechanism.

To model the onset of this prototypical reaction, we located a negative point charge of 
-0.1 a.u. at $3 \AA$ from the carbon of the alkyl halide and aligned with the $\mathrm{C}-\mathrm{Cl}$ bond. The perturbed wave function has been computed and the electron density deformation calculated. The latter is represented in Fig. 2.

Figure 2: Electron density response for $\mathrm{CH}_{3} \mathrm{Cl}$ perturbed by a -0.1 a.u. point charge placed at $3 \AA$ from the $\mathrm{C}$ atom. Surfaces colour scheme: yellow, electron density depletion $(\delta \rho(\mathbf{r})<0)$; red, electron density relocation $\delta \rho(\mathbf{r})>0$ Isodensity: $1.10^{-4}$ a.u. Atom colour scheme: H, white; C, gray; $\mathrm{Cl}$, green.

As expected, along the $\mathrm{C}-\mathrm{Cl}$ bond the electron density is shifted from the carbon to the chlorine. This distorted electron density is principally reached by the promotion of a tiny fraction of electron from the HOMO-3 $(\sigma \mathrm{C}-\mathrm{Cl}$ orbital $)$ to the LUMO $\left(\sigma^{\star} \mathrm{C}-\mathrm{Cl}\right)$. The transition density that triggers this excitation is represented in Fig. 3. It may be noted already that this transition density connects the ground and 16th excited states, and not as could be naïvely expected the ground and first excited state. The fact that excited state number 16 contributes more significantly than the first 15 ones can be understood from Equation 17: the weight of a given transition density will be given by the ratio of an "overlap" term of the transition density and the perturbation, and of the excitation energy. Here, the "overlap" term in excited state number 16 is thus very large, compensating the 
non negligible excitation energy.

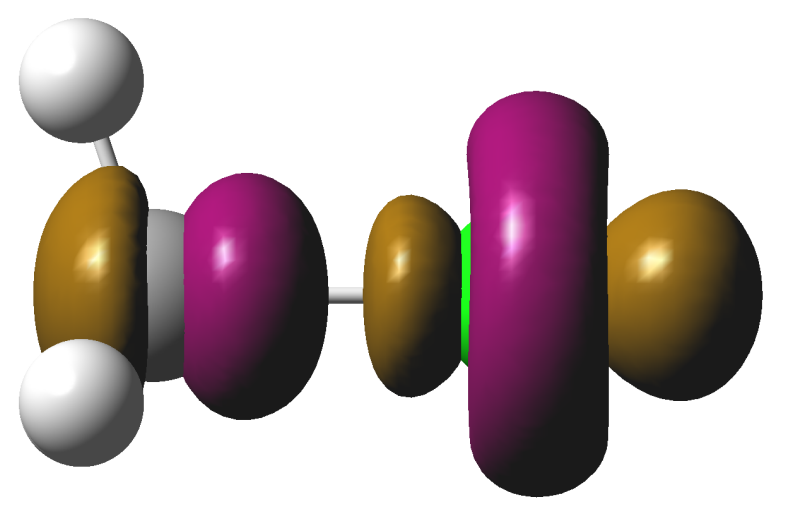

Figure 3: $16^{\text {th }}$ methylchloride transition density, represented at an isovalue of $1.10^{-3}$ a.u. Colour scheme: purple, $\rho_{0}^{k}(\mathbf{r})<0$; brass, $\rho_{0}^{k}(\mathbf{r})>0$.

The perturbation consequence is thus principally the population of the anti-bonding $\sigma^{\star} \mathrm{C}-\mathrm{Cl}$, that weakens the carbon chlorine bond. Aside from this, we may also note an electron density reorganisation occurring in the orthogonal direction with respect to the attack axis, noticeably a relocation basin in the vicinity of the $\mathrm{C}-\mathrm{H}$ bonds. A more careful examination reveals that electrons relocate essentially on the side of the $\mathrm{C}-\mathrm{H}$ bonds which is the furthest away from the perturber. This relocation of electrons should in principle lead to a displacement of the $\mathrm{H}$ atoms away from the perturber, consistently with the expected nuclei motion along the reaction path (Walden inversion). The depletion on the $\mathrm{Cl}$ atom can then be conceived as "the lone pair electrons making room for the C-H electrons to relocate". We thus appear to retrieve all the expected features of the $\mathrm{SN}_{2}$ reaction.

To check the validity of our conclusions, we then performed a geometry optimisation of $\mathrm{CH}_{3} \mathrm{Cl}$ in the presence of an explicit -0.1 a.u. point charge fixed at $3 \AA$ from the $\mathrm{C}$ atom. In 
these conditions, the C-Cl bond length increases from $1.7946 \AA$ to $1.8100 \AA$ (0.9\% increase), while at the same time the $\mathrm{ClCH}$ angles drop from $108.37^{\circ}-108.39^{\circ}$ to $106.77^{\circ}-106.82^{\circ}$ (relative variation of around $1.4 \%$ ). The nuclei motions are thus in complete agreement with those predicted from the $\delta \rho(\mathbf{r})$ calculation.

Therefore, contrarily to what is generally assumed, we may state that it is the electrostatic potential created by the charge borne by the nucleophile that initiates the bond breaking between the carbon and the halide in the prototypical $\mathrm{SN}_{2}$ reaction. The electrostatic field induced by the nucleophile during its approach prepares the substrate for the reaction to come through three processes: i) the carbon atom becomes more and more electrophilic as it looses electron density; ii) the promotion of a fraction of electron into the LUMO initiates the $\mathrm{C}-\mathrm{Cl}$ bond cleavage, or said otherwise the transfer of this fraction of electron to $\mathrm{Cl}$ leads this atom to enhance its $\mathrm{Cl}^{-}$character (leaving group ability); iii) the relocation of electrons on the sides of the $\mathrm{C}-\mathrm{H}$ bonds leads the $\mathrm{H}$ atoms to move away from the nucleophile, eventually reaching the Walden inversion. It can be surmised that the polarising and dissociating effects of water as a solvent stem from the same phenomenon.

\section{Electrophilic assistance}

The so-called Electrophilic assistance is a phenomenon that can be found in the reactivity of carbonyl compounds. It characterises the enhancement of the carbonyl carbon atom reactivity with respect to a nucleophile when the carbonyl oxygen is either coordinated to a metal or bonded to a proton (protonated). Generally, this reactivity improvement is explained by an increase in the oxygen electronegativity due to its interaction with the electrophile (metal or proton), which increases the $\pi^{\star}(\mathrm{C}-\mathrm{O})$ orbital asymmetry. This $\pi^{\star}(\mathrm{C}$ O) being the LUMO, a larger lobe and thus a larger electrophilicity is found on the carbonyl carbon.

From this discussion it appears the electrophilic assistance can also be conceived as originating in the electrostatic potential induced by the electrophile on the carbonyl. One 
could thus expect to characterise this effect through a calculation of the responses of a carbonyl compound to a perturbation by a positive point charge. To check this, we calculated the response of a formaldehyde molecule to a +0.05 u.a. point charge perturbation. The perturber is located roughly along the $\mathrm{C}-\mathrm{O}$ bond and at $3 \AA$ from the oxygen atom. Note that a series of calculation revealed that the responses main features remained qualitatively unchanged when the location (at constant distance) of the point charge was modified. The density polarisation is represented in Fig. 4.

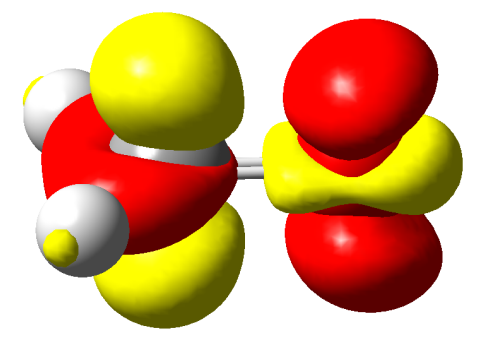

Figure 4: Electron density response for formaldehyde perturbed by a +0.05 a.u. point charge placed at $3 \AA$ from the $\mathrm{O}$ atom, represented by the hydrogen atom. Surfaces colour scheme: yellow, electron density depletion $(\delta \rho(\mathbf{r})<0)$; red, electron density relocation $\delta \rho(\mathbf{r})>0$ Isodensity: $1.10^{-4}$ a.u. Atom colour scheme: $\mathrm{H}$, white; $\mathrm{C}$, gray; $\mathrm{O}$, red.

On the one hand, it is plain to see that as expected the $\pi$ system is more polarized than the $\sigma$ system. According to the density polarisation sign, the electron flows from the carbon towards the oxygen. This $\pi$ electron reshuffling is the consequence of the promotion of a small fraction of electron of the $\pi(\mathrm{C}-\mathrm{O})$ bonding orbital to the $\pi^{\star}(\mathrm{C}-0)$ anti-bonding orbital. 
The transition density that enables this electron excitation is represented in Fig. 5

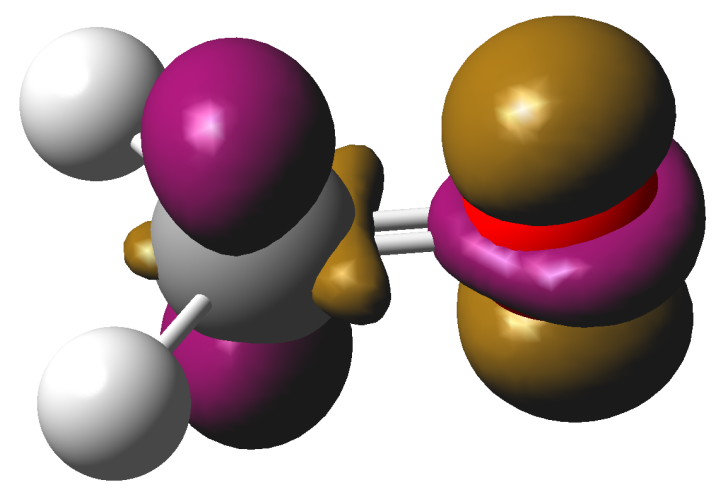

Figure 5: $7^{\text {th }}$ formaldehyde transition density, represented at an isovalue of $1.10^{-3}$ a.u. Colour scheme: purple, $\rho_{0}^{k}(\mathbf{r})<0$; brass, $\rho_{0}^{k}(\mathbf{r})>0$.

On the other hand, what was less expected when one looks at Fig 4 is the presence and direction of the $\sigma$ polarisation density. Indeed, according to the electron polarisation sign, there is a back donation from the oxygen atom toward the carbon through the $\sigma$ backbone, just as if the $\sigma$ electron system tries to counterbalance the $\pi$ reorganisation. This could be seen as a molecular equivalent of either the Lenz Law of magnetic induction or the Le Chatelier principle in chemistry. It may also be noted that a similar effect was already present in the previous example, with opposite and orthogonal electron polarisations borne by the same groups.

Again to check whether the predictions are correct and if indeed the C-O bond is weakened by the presence of a point charge, a geometry optimization has been run. For a point charge of +0.5 a.u. located at $3 \AA$ from the oxygen and aligned with the carbonyl group, the C-O bond length increases from $1.1987 \AA$ to $1.2048 \AA$. One also observes a small decrease 
of the $\mathrm{C}-\mathrm{H}$ bond lengths that suggests $\mathrm{CH}$ bonds are strengthened. This could actually be inferred from Fig. 4: as the carbon overall loses electrons, its electronegativity is expected to increase and should thus become closer to that of hydrogen (Pearson values: $\chi(H)=7.18 \mathrm{eV}$, $\chi(C)=6.27 \mathrm{eV})^{26}$ making the $\mathrm{C}-\mathrm{H}$ bonds stronger.

\section{The trans effect in an octahedral complexes}

In the last two illustrations we focused on examples from the field of organic chemistry. Stating the obvious, the proposed approach here is not restricted to this sole area of chemistry. To illustrate this point, we propose in this subsection to revisit a well-known effect in coordination chemistry: the trans effect in octahedral complexes. Let us consider the following complex: $\left[\mathrm{Co}\left(\mathrm{NO}_{2}\right)_{3}\left(\mathrm{CH}_{3}\right)\left(\mathrm{NH}_{3}\right)_{2}\right]^{-} \cdot{ }^{27}$ Experimentally, it presents a markedly elongated Conitrite bond trans to the methyl ligand $(+0.10 \AA)$. In previous studies, ${ }^{28,29}$ we showed that this elongation could be related to a marked difference of electrophilicity on the coordination position, probed through a dual descriptor calculation on fragments of the complex (where one nitrite, either cis or trans to methyl, was removed). A large reduction of electrophilicity could be observed on the trans position with respect to methyl, consistent with a weaker coordination of a ligand on this position and thus an elongation of the Co-nitrite bond. ${ }^{\mathrm{c}}$

We give in Table 1 and on Figure 6 the outcome of a $E^{(2)}, \delta \rho(\mathbf{r})$ and $\delta N$ calculation for the same coordination-deficient fragments, undergoing a perturbation by a -0.01 a.u. point charge placed at the position the missing ligands occupy in the full complex. $\delta \rho(\mathbf{r})$ main features can also be linked to a single transition density in both cases, as represented on Figure 7. However, it must be noted that in this case it is much harder to attribute this transition density to a small number of electron transfers between MOs, as excited states decompose over a rather large number of $\mathrm{MO} \rightarrow \mathrm{MO}$ excitations. Nevertheless, the shape of $\delta \rho(\mathbf{r})$ suggests in both cases that a significant part of the response is due to the excitation of a fraction of electron from the M-L $\sigma$-bond trans to the vacancy, to other (orthogonal) M-L

\footnotetext{
${ }^{\mathrm{c}}$ Note that this electrophicility reduction is also apparent from the values of the global electrophilicity $\omega=\mu / \eta^{2}$, which equals $6.5 \mathrm{eV}$ for the trans deprived complex and $8.7 \mathrm{eV}$ for the cis fragment.
} 

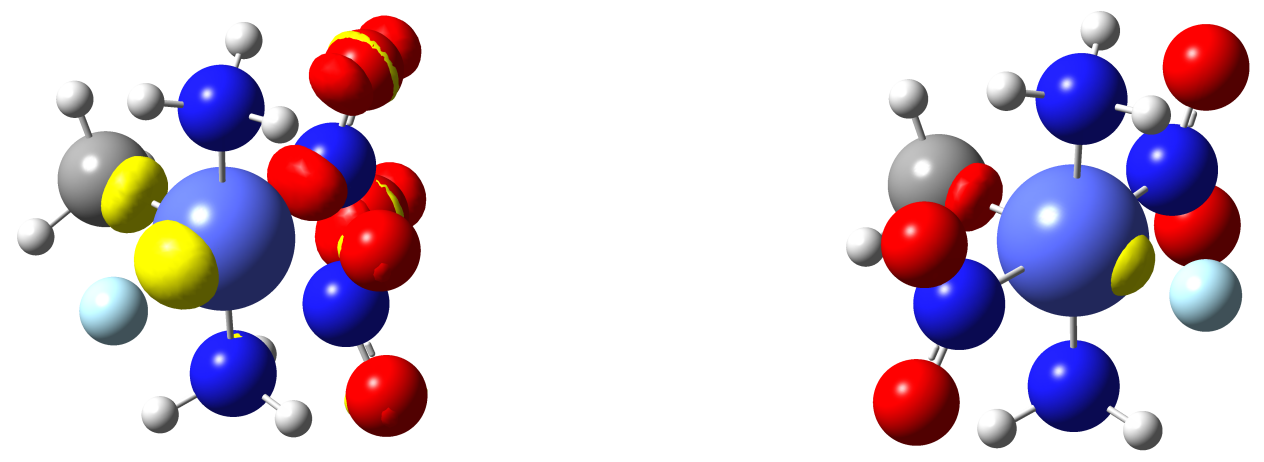

Figure 6: Electron density response for fragment complexes derived from $\left[\mathrm{Co}\left(\mathrm{NO}_{2}\right)_{3}\left(\mathrm{CH}_{3}\right)\left(\mathrm{NH}_{3}\right)_{2}\right]^{-}$by the abstraction of one nitrite ligand on the cis (left) or trans (right) position, perturbed by a -0.05 a.u. point charge placed at the coordination vacancies. Same surface colour scheme as previously, isodensity: $1.10^{-4}$ a.u. Atom colour scheme: H, white; C, gray; $\mathrm{N}$, royal blue; O, red; Co, lavender.

centred MOs.

From Table 1, the system responses are much weaker on the trans position than on the cis one. The trans coordination position thus appears largely less polarisable than the cis, resulting in a significantly smaller energy stabilisation upon the approach of a perturbing point charge on this position. We thus retrieve a tendency of the system to decrease coordination on the trans position compared to cis.

Table 1: Polarisation energy and number of shifted electrons for perturbations by a $\mathrm{q}=-0.01$ a.u. point charge on coordination vacancies of Co complex. The absolute $E^{(2)}$ and $\delta N$ values are given, as well as the normalised $E^{(2)} / q^{2}$ and $\delta N / q$ values (in italics).

\begin{tabular}{l|r|r} 
location & $E^{(2)}$ & $\delta N$ \\
\hline cis & $-40.2 \mu \mathrm{eV}$ & $0.0016 \mathrm{e}$ \\
& $-0.402 \mathrm{eV} / \mathrm{e}^{2}$ & $0.16 \mathrm{e} / \mathrm{e}$ \\
\hline trans & $-22.9 \mu \mathrm{eV}$ & 0.0009 \\
& $-0.229 \mathrm{eV} / \mathrm{e}^{2}$ & $0.09 \mathrm{e} / \mathrm{e}$ \\
\hline
\end{tabular}

At this stage, one may rightfully object that the smaller response could merely be due to the significant elongation of the coordination bond on the trans position: pushing the point charge away, the electrostatic potential experienced by the molecule should indeed be 

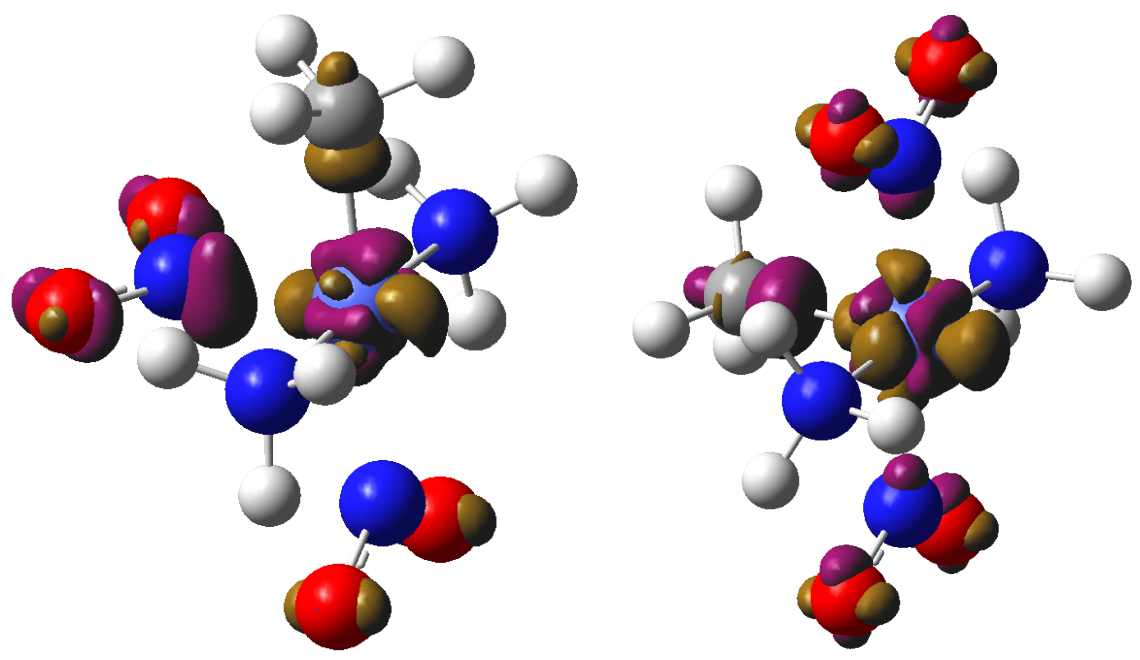

Figure 7: 33th and 22th transition densities, respectively, for the cis- (left) and trans nitritedeprived fragments deriving from $\left[\mathrm{Co}\left(\mathrm{NO}_{2}\right)_{3}\left(\mathrm{CH}_{3}\right)\left(\mathrm{NH}_{3}\right)_{2}\right]^{-}$, represented at an isovalue of $1.10^{-3}$ a.u. Colour scheme: purple, $\rho_{0}^{k}(\mathbf{r})<0$; brass, $\rho_{0}^{k}(\mathbf{r})>0$.

reduced.

Actually, this relates to a larger issue of the proposed approach so far: the perturber was in all cases placed according to our understanding of the rules of chemistry. The positions were thus quite arbitrary, in the sense that they did not stem directly from the formalism.

In the case of the Co complex, a possible way to get rid of this "knowledge induced bias" would be a partial alchemical change, ${ }^{30-33}$ that is to place the point charge perturbation directly at the Co nuclei position, for both coordination deficient fragments. Indeed, no assumption would thus be made about the actual location of the missing ligands. Interestingly, although this leads to major changes in the shape of the electron density distortion, the same tendency is retrieved in the values of $E^{(2)}$ and $\delta N$ : the responses on the cis position $(-1.87$ $\left.\mathrm{eV} / \mathrm{e}^{2}, 0.40 \mathrm{e} / \mathrm{e}\right)$ are larger than those on the trans position $\left(-1.40 \mathrm{eV} / e^{2}, 0.30 \mathrm{e} / \mathrm{e}\right)$. The larger polarisability of the electron density on the cis position is thus clearly retrieved, and is an intrinsic feature of these fragment complexes.

Taking inspiration from these last results, we then wondered whether we could obtain an even more general picture, by calculating the response of the whole complex to a small perturbation exerted directly on the ligand positions (at the $\mathrm{N}$ nuclei). Placing a -0.1 a.u. 
point charge either on the cis or trans $\mathrm{N}$ atoms of the corresponding nitrite ligands, we obtained the data presented in Table 2 .

Table 2: Polarisation energy and number of shifted electrons for perturbations by a $\mathrm{q}=-0.1$ a.u. point charge on the cis and trans nitrite $\mathrm{N}$ atoms of a Co complex. The absolute $E^{(2)}$ and $\delta N$ values are given, as well as the normalised $E^{(2)} / q^{2}$ and $\delta N / q$ values (in italics).

\begin{tabular}{l|r|r} 
location & $E^{(2)}$ & $\delta N$ \\
\hline cis & $-7.42 \mathrm{meV}$ & $0.0167 \mathrm{e}$ \\
& $-0.742 \mathrm{eV} / \mathrm{e}^{2}$ & $0.167 \mathrm{e} / \mathrm{e}$ \\
\hline trans & $-13.78 \mathrm{meV}$ & 0.0237 \\
& $-1.378 \mathrm{eV} / \mathrm{e}^{2}$ & $0.237 \mathrm{e} / \mathrm{e}$ \\
\hline
\end{tabular}

As one can note, we obtain larger responses when the perturbation is exerted on the trans position. At first glance, these results seem to contradict the previous ones. Actually, they are fully consistent with the expected trends: the trans position is in principle associated to a weaker coordination of the nitrite ligand. Thus, the ligand in this position is expected to be more reactive than the cis one. Said otherwise, the trans nitrite ligand should behave more like a "free nitrite species" than the cis one, and should thus likely end up being more polarisable.

From these last results, we are now able to conceive a general methodology to explore chemistry using our perturbation framework: instead of placing perturbing point charges on specific locations (proposed on the basis of our chemical culture and thus likely subject to bias), we may directly probe the response of the system via nuclei-centred perturbations. It may be noted that in all cases, despite the fact that the perturbation is placed at nuclei position, the electron density response is principally driven by valence electrons and orbitals (and not core electrons as could have been presumed). This is rather satisfactory in the light of the usual models of chemistry, especially the Slater's rules. ${ }^{34}$

\section{Nucleophilic additions on $\alpha, \beta$-conjugated carbonyls}

In this last subsection, we would like to illustrate the potential of the proposed descriptors to offer a different perspective on the origin of chemical selectivity. We propose here to focus 
on the case of the addition of nucleophiles to $\alpha, \beta$-conjugated carbonyls.

Using simple arguments (such as mesomeric effects), one may easily show that both the carbonyl (2) and terminal alkene (4) C atoms are electrophilic, and thus susceptible to react (atom numbering is given in Fig. 8). From an experimental perspective, it appears

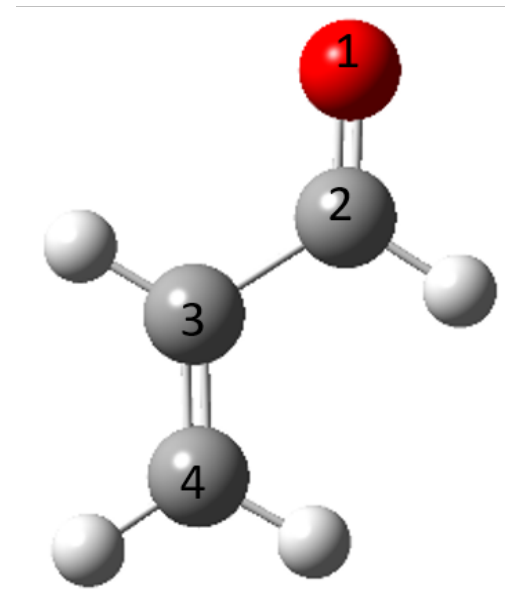

Figure 8: Atom numbering for acrolein.

that nucleophilic addition on both sites are feasible. Hard nucleophiles lead principally to the formation of the $(1,2)$ product, while soft nucleophiles lead preferentially to the $(1,4)$ addition. This is often explained on the basis of the charge versus orbital control (KlopmanSalem) $)^{2,3}$ : while the most positive atomic charge is found on $\mathrm{C} 2$, orbital-based descriptors (either coefficients in the LUMO or electrophilic superdelocalisability index) indicate that $\mathrm{C} 4$ is the most reactive site.

Equivalently, we may state that $\mathrm{C} 2$ is a harder electrophile than $\mathrm{C} 4$, or that $\mathrm{C} 2$ is less polarisable than $\mathrm{C} 4$. Thus, provided that both atoms would experience the same perturbation, we would expect $\mathrm{C} 4$ to exhibit a significantly larger response (either in polarisation energy or number of shifted electrons) than C2. Calculations on an acroleine molecule are indeed consistent with these predictions. Placing a -0.05 a.u. point charge perturbation on either $\mathrm{C}$ nuclei, we first of all observe that the electron density responses, as displayed on Figure 9 , are principally borne by the $\pi$-system of the molecule, in complete line with our previous observations. 

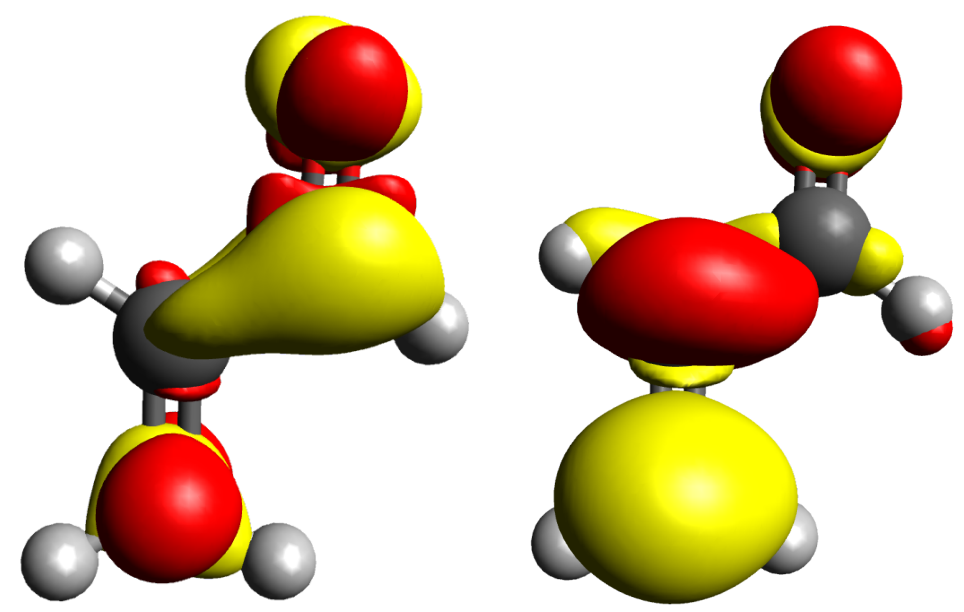

Figure 9: Electron density response for an acroleine molecule perturbed by a -0.05 a.u. point charge placed at the $\mathrm{C} 2$ position (left) and C4 position (right). Same surfaces and atom colour schemes as previously. Isodensity: $2 \cdot 10^{-4}$ a.u.

In both cases, a few transition densities contribute significantly to $\delta \rho(\mathbf{r})$, especially transitions number 3 and 12 when perturbation is exerted on position 2, and transitions number 3 and 8 on $\mathrm{C} 4$.

A schematic representation of these three main transitions and the principal MO-based electron transfers they involve is given on Fig. 10. It is easily seen that transition number 3 should result in the $\mathrm{C} 3-\mathrm{C} 4 \pi$ bond breaking while transition number 12 accounts for the $\mathrm{C}(2)-\mathrm{O}(1) \pi$ bond breaking. Obviously, transition number 3 is associated to a much lower energy than transitions 8 and 12, and is thus more accessible to the system.

On a quantitative point of view, we observe a significantly stronger response when the perturbation occurs at $\mathrm{C} 4$, as can be seen from Table 3 or Figure 9. Noteworthy, polarisation energy is nearly three times stronger on $\mathrm{C} 4$ compared to $\mathrm{C} 2$. Thus, we completely reproduce the more pronounced softness of $\mathrm{C} 4$.

\section{Conclusion}

In this publication, we were interested in developing new reactivity descriptors, relying explicitly on a perturbative development. Using Rayleigh-Schrödinger perturbation theory 

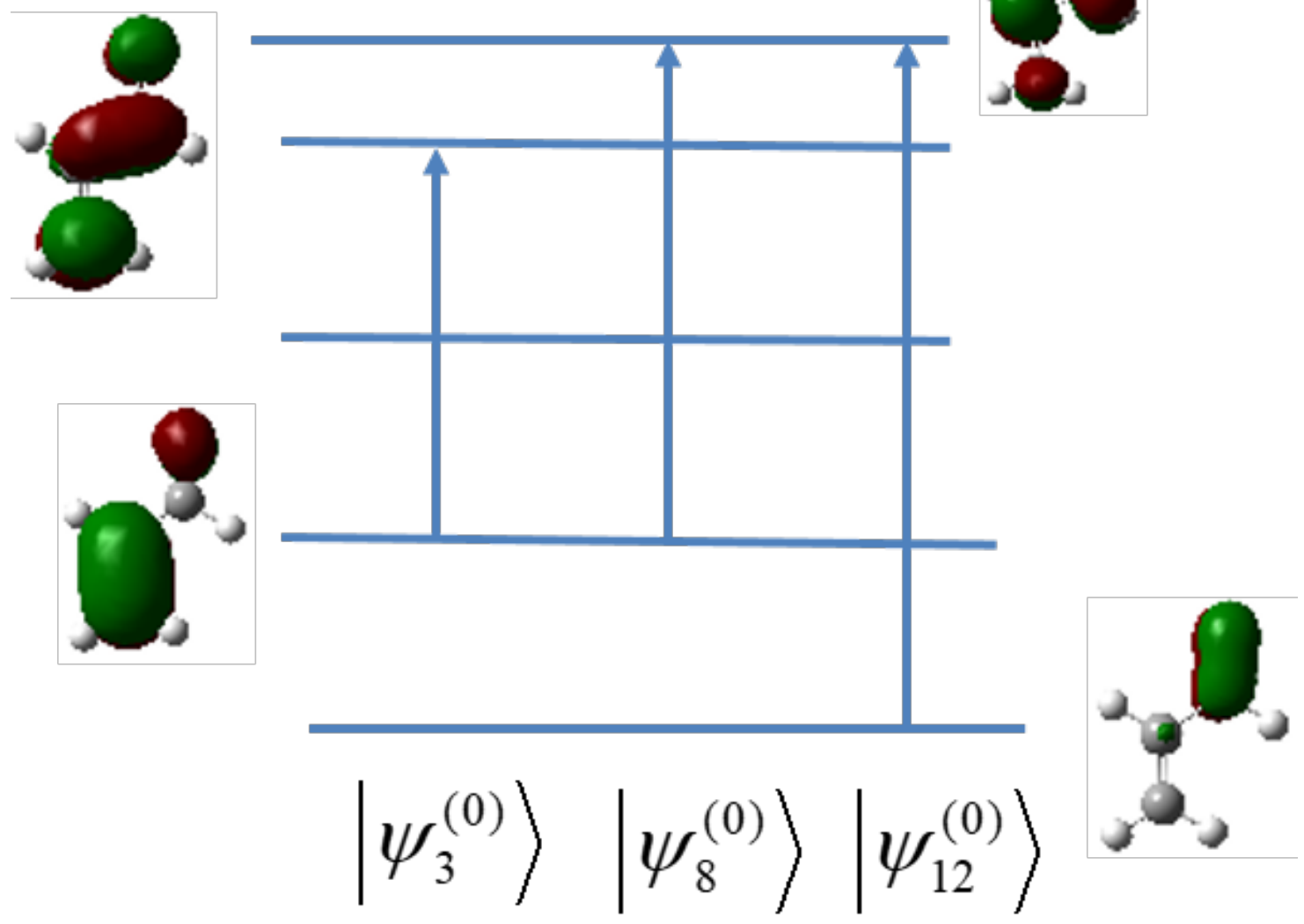

Figure 10: Main contributing transitions to the density polarization, and representation of the MOs they involve.

within a (time-dependant) Density Functional Theory framework, we derived and studied three reactivity descriptors, related to the responses of a molecular system to an electrostatic (point-charge) perturbation. We here focused on the calculation of $\delta \rho(\mathbf{r})$, the first order reorganisation of electron density, $\delta N$, the total number of shifted electron under perturbation at first order, and $E^{(2)}$, the second-order correction to energy. Through identification of these descriptors to quantities deriving from the linear response function, a well-known Conceptual-DFT descriptor, and physical interpretation of the equations, we could show the proposed descriptors should convey information on reactivity and selectivity, both at a qualitative and quantitative level. Application of these descriptors on different systems whose chemical properties are known indeed proved their strong predictive potential. In all cases $\delta \rho(\mathbf{r})$ is able to provide qualitative information about the fate of the studied system, 
Table 3: Polarisation energy and number of shifted electrons for perturbations by a -0.05 a.u. point charge on the $\mathrm{C}$ atoms of an acroleine molecule. The absolute $E^{(2)}$ and $\delta N$ values are given, as well as the normalised $E^{(2)} / q^{2}$ and $\delta N / q$ values.

\begin{tabular}{l|r|r} 
location & $E^{(2)}$ & $\delta N$ \\
\hline $\mathrm{C} 2$ & $-1.90 \mathrm{meV}$ & $0.008 \mathrm{e}$ \\
& $-0.76 \mathrm{eV} / \mathrm{e}^{2}$ & $0.16 \mathrm{e} / \mathrm{e}$ \\
\hline $\mathrm{C} 4$ & $-5.40 \mathrm{meV}$ & $0.015 \mathrm{e}$ \\
& $-2.16 \mathrm{eV} / \mathrm{e}^{2}$ & $0.30 \mathrm{e} / \mathrm{e}$ \\
\hline
\end{tabular}

indicating the expected electron flows and hinting about the subsequent nuclei motions. $\delta N$ and $E^{(2)}$, on the other hand, allow a more quantitative insight on reactivity, allowing for instance to characterise numerically which site in a molecule is most prone to react.

Interestingly, as we could show in the last two examples, the proposed formalism also allows to obtain insights on reactivity without the need for the user to implement his/her own chemical knowledge. Indeed, perturbation on nuclei still induces an electronic response relying on the "valence shell". In turn, this allows to conceive a full automation of the reactivity characterisation of a given system, through a systematic evaluation of the descriptors after perturbation on each nucleus. We feel this offers an interesting perspective to develop in the close future. The bridge with conceptual DFT also offers interesting perspectives; noticeably, what could be the equivalent of our descriptors within the grand-canonical ensemble?

On a different viewpoint, we could also use our approach to evaluate reactivity in confined systems, for instance within enzymatic cavities or within a solid catalyst. At a first level of modelling, the confining entities could indeed be simply modelled by a collection of point charges, inducing an electrostatic potential on the chemical reagents they embed. One may then wonder how this embedding potential affects reactivity: could this electrostatic perturbation play a significant (if not preponderant) role in the observed catalyses? 


\section{Acknowledgments}

F.D.P. wishes to acknowledge the Vrije Universiteit Brussel (VUB) for a Strategic Research Program and the Francqui foundation for a position as Francqui research professor.

\section{References}

(1) Pearson, R. G. Journal of the American Chemical Society 1963, 85, 3533.

(2) Salem, L. Journal of American Chemical Society 1968, 90, 543-552.

(3) Klopman, G. Journal of American Chemical Society 1968, 90, 223-234.

(4) Fukui, K.; Fujimoto, H. Bulletin of the Chemical Society of Japan 1968, 41, 1989-1987.

(5) Liu, R. S. H. Journal of Chemical Education 2002, 79, 183.

(6) Walsh, A. D. Trans. Faraday Soc. 1949, 45, 179-190.

(7) Pearson, R. G. J. Am. Chem. Soc. 1988, 110, 2092-2097.

(8) Pearson, R. G. Accounts of Chemical Research 1993, 26, 250-255.

(9) Nagy, Á. Journal of Chemical Sciences 2005, 117, 437-440.

(10) Fukui, K. Science 1982, 218, 747-754.

(11) Tognetti, V.; Morell, C.; Ayers, P. W.; Joubert, L.; Chermette, H. Phys. Chem. Chem. Phys. 2013, 15, 14465-14475.

(12) De Proft, F.; Forquet, V.; Ourri, B.; Chermette, H.; Geerlings, P.; Morell, C. Physical Chemistry Chemical Physics 2015, 17.

(13) Schrödinger, E. Annalen der Physik 1926, 385, 437-490. 
(14) Ayers, P. W.; Parr, R. G. Journal of the American Chemical Society 2000, 122, 20102018.

(15) Geerlings, P.; Fias, S.; Boisdenghien, Z.; De Proft, F. Chem. Soc. Rev. 2014, 43, 49895008.

(16) Sablon, N.; Proft, F. D.; Geerlings, P. The Journal of Physical Chemistry Letters 2010, 1, 1228-1234.

(17) Boisdenghien, Z.; Fias, S.; Van Alsenoy, C.; De Proft, F.; Geerlings, P. Physical chemistry chemical physics : PCCP 2014, 16, 14614-24.

(18) Boisdenghien, Z.; Van Alsenoy, C.; De Proft, F.; Geerlings, P. Journal of Chemical Theory and Computation 2013, 9, 1007-1015, PMID: 26588743.

(19) Boisdenghien, Z.; Fias, S.; Pieve, F. D.; Proft, F. D.; Geerlings, P. Molecular Physics 2015, 113, 1890-1898.

(20) Sablon, N.; Proft, F. D.; Geerlings, P. Chemical Physics Letters 2010, 498, $192-197$.

(21) Naray-Szabo, G.; Ferenczy, G. G. Chemical Reviews 1995, 95, 829-847.

(22) Neese, F. Wiley Interdiscip. Rev.: Comput. Mol. Sci. 2012, 2, 73-78.

(23) Hirata, S.; Head-Gordon, M. Chemical Physics Letters 1999, 314, 291 - 299.

(24) Carey, F. A.; Sundberg, R. J. Advanced Organic Chemistry part A; Springer, 2007.

(25) Anslyn, E. V.; Dougherty, D. A. Modern Physical Organic Chemistry; University Science Books, 2006; Chapter 11.

(26) Parr, R. G.; Yang, W. Density-Functional Theory of Atoms and Molecules; International Series of Monographs on Chemistry; Oxford University Press, 1994. 
(27) Kofod, P.; Harris, P.; Larsen, S. Inorganic Chemistry 1997, 36, 2258-2266, PMID: 11669859.

(28) Guégan, F.; Tognetti, V.; Joubert, L.; Chermette, H.; Luneau, D.; Morell, C. Phys. Chem. Chem. Phys. 2016, 18, 982-990.

(29) Tognetti, V.; Guégan, F.; Luneau, D.; Chermette, H.; Morell, C.; Joubert, L. Theoretical Chemistry Accounts 2017, 136, 85.

(30) von Lilienfeld, O. A.; Tuckerman, M. E. The Journal of Chemical Physics 2006, 125, 154104.

(31) von Lilienfeld, O. A.; Lins, R. D.; Rothlisberger, U. Phys. Rev. Lett. 2005, 95, 153002.

(32) Muñoz, M.; Cárdenas, C. Phys. Chem. Chem. Phys. 2017, 19, 16003-16012.

(33) Balawender, R.; Lesiuk, M.; De Proft, F.; Van Alsenoy, C.; Geerlings, P. Phys. Chem. Chem. Phys. 2019, --

(34) Slater, J. C. Phys. Rev. 1930, 36, 57-64. 